\title{
OCORRÊNCIA DE Sarconesia chlorogaster (WIEDEMANN, 1830), EM CRIAÇÃO DE BOVINOS LEITEIROS, NO SUL DO RIO GRANDE DO SUL, BRASIL
}

\author{
PINTO, Diego Moscarelli; \\ AGUIAR, Cíntia Lidiane Guidotti \\ ALVES, Bruna Farias ${ }^{3}$; \\ TORRES, Maria Izabel de Tourinho ${ }^{3}$; \\ DANELUZ, Marina Oliveira ${ }^{3}$; \\ MARTINS, Natalia Soares ${ }^{3}$; \\ BECKER, Marcelo ${ }^{4}$; \\ JUNIOR, Altair Aguiar ${ }^{5}$
}

${ }^{1}$ Professor Adjunto do Departamento de Veterinária Preventiva/UFPEL. ${ }^{2}$ Bióloga, Mestre pelo Programa de PósGraduação em Veterinária/UFPEL. ${ }^{3}$ Aluna de Graduação em Veterinária/UFPEL. ${ }^{4}$ Aluno de Graduação em Veterinária/UNIPAMPA. ${ }^{5}$ Administrador, Estagiário LADOPAR/UFPEL.

RESUMO

$\mathrm{E}$ ste estudo teve o objetivo de fornecer informações sobre o comportamento de Sarconesia chlorogaster em criação de gado leiteiro na região sul do estado do Rio Grande do Sul. O trabalho foi realizado no Centro Agropecuário da Palma, pertencente à Universidade Federal de Pelotas (UFPEL). Para a captura dos califorídeos foram utilizadas duas armadilhas do tipo W.O.T. (Wind Oriented Trap), sendo estas iscadas com $250 \mathrm{~g}$ de fígado bovino em decomposição. As armadilhas foram expostas a 50 metros do local de ordenha durante sete dias por mês ao longo de 12 meses, entre o 10 o e 20 dias e os espécimes capturados foram retirados das armadilhas e levados para o Laboratório de Biologia de Insetos para triagem, contagem e identificação. Durante o período de março de 2007 a fevereiro de 2008, foram capturados 73 exemplares de S. chlorogaster, sendo que novembro foi o mês de maior captura (53) e março o de menor captura (01). Foi constatada uma oscilação na flutuação populacional deste califorídeo, influenciada principalmente pela variação das temperaturas. A ocorrência de $S$. chlorogaster em criação de gado leiteiro, na região sul do Rio Grande do Sul, é influenciada principalmente pela temperatura.

Palavras-chave: Armadilha. Comportamento. Calliphoridae. 


\section{INTRODUÇÃO}

A espécie Sarconesia chlorogaster (Wiedemann, 1830) pertence à família Calliphoridae, subfamília Toxotarsinae. Essa subfamília é endêmica da região Neotropical e sua distribuição geográfica ocorre entre $4^{\circ} \mathrm{S}$ e $40^{\circ} \mathrm{S}$, em regiões de clima frio e em altitudes que variam entre 0 e 4200 metros. S. chlorogaster tem distribuição exclusivamente sul americana, com registros conhecidos para a região sul do Brasil (Paraná e Rio Grande do Sul) (CARVALHO; RIBEIRO, 2000), Argentina, Uruguai, e as regiões de altitude próxima a 1000 metros de Bolívia, Peru e Chile (DEAR, 1979; JAMES, 1970).

As moscas da família Calliphoridae têm importante função na natureza, participando da cadeia alimentar e da reciclagem da biomassa. Atuam como agentes mecânicos e/ou biológicos, (CARVALHO et al., 2003; FURUSAWA; CASSINO, 2006), veiculando diversos agentes patogênicos que causam enfermidades parasitárias ao homem e aos animais domésticos. Além disso, podem ser utilizadas em terapia larval que é um tipo de bioterapia que envolve a introdução intencional de larvas vivas desinfectadas em tecidos humanos ou animais feridos (como a pele) com a proposta de seletivamente limpar somente o tecido necrótico da ferida, de modo a melhorar a cura. Isso ocorre, pois as larvas se alimentam somente do tecido necrótico (SHERMAN et al., 2000).

No sul do Brasil ocorrem cerca de 20 espécies de Calliphoridae, sendo que no Rio Grande do Sul, as mais prevalentes são Cochliomyia hominivorax (Coquerel, 1858) e Chrysomya megacephala (Fabricius, 1794); Chrysomya albiceps (Wiedemann, 1819); Chrysomya putoria (Wiedemann, 1818); Sarconesia chlorogaster (Wiedemann, 1831) (AZEVEDO, 2006; CARVALHO; RIBEIRO, 2000).

Do ponto de vista médico-sanitário, há uma crescente preocupação epidemiológica com esse grupo de insetos, dada a sua grande capacidade de dispersão, elevada densidade populacional e diversificado hábito alimentar, aliados ao alto grau de sinantropia e endofilia, tornando-os potenciais transmissores de agentes enteropatogênicos ao homem e animais domésticos (FURLANETTO et al., 1984; LAOS et al., 2004; MALDONADO; CENTENO, 2003; MARILUIS et al., 1989). 
S. Chlorogaster apresenta ciclo biológico holometabólico, com fases de ovo, larva, pupa e adulto, em que as larvas se caracterizam por seus hábitos alimentares necrófagos, e os adultos por sua preferência por áreas urbanas e seu elevado grau de sinantropia, o que permite sua utilização como indicadora do intervalo pós-morte em entomologia médicocriminal (CARVALHO; MELLO-PATIU, 2008; MOURA; BONATTO, 1999).

Vários autores têm estudado o ciclo de vida desse califorídeo em laboratório, visando entender melhor o comportamento destes insetos e têm verificado que a temperatura e umidade são fatores importantes principalmente no desenvolvimento das formas imaturas (BONATTO, 1996; GREENBERG; SZYSKA, 1984; KRÜGER et al., 2010; QUEIROZ et al., 1985). Entretanto, poucos são os trabalhos que tratam do comportamento das populações nos locais de criação de animais.

Dada a importância desse califorídeo, bem como a pouca literatura sobre o assunto, realizou-se este estudo com o objetivo de fornecer informações sobre a ocorrência e comportamento populacional de S. chlorogaster em local de criação de gado leiteiro, no sul do Rio Grande do Sul.

\section{MATERIAL E MÉTODOS}

O trabalho foi realizado no Centro Agropecuário da Palma com 1.256 ha de área, pertencente à Universidade Federal de Pelotas (UFPEL), localizado no município de Capão do Leão, RS, a quinze km da cidade de Pelotas, RS, à margem da BR 116, no km 535, cujas coordenadas geográficas são $31^{\circ} 52^{\prime} 00^{\prime \prime}$ de latitude Sul e $52^{\circ} 21^{\prime} 24^{\prime \prime}$ de longitude Oeste.

O período experimental foi de doze meses, entre março de 2007 e fevereiro de 2008. Para a captura dos califorídeos, empregaram-se duas armadilhas do tipo WOT (Wind oriented trap - armadilha orientada pelo vento).

As armadilhas foram confeccionadas artesanalmente de acordo com o modelo de Broce et al. (1977), modificado por Oliveira (1980). Cada armadilha constituiu-se de um balde plástico de cor amarela com capacidade para oito litros, sendo removido o fundo, adaptando-se uma peneira plástica removível, que permitia a retirada dos espécimes coletados e também a 
colocação do recipiente com a isca. $\mathrm{Na}$ alça do balde foi anexada uma aleta de metal (alumínio) para direcionar a abertura do balde no sentido do vento. Na borda do balde, colocou-se um telado plástico com uma pequena abertura, tipo cone invertido, que permitia a entrada dos espécimes na armadilha.

$\mathrm{Na}$ parte inferior da armadilha, colocaram-se recipientes que funcionavam como contrapesos para evitar o movimento de pêndulo. As armadilhas foram amarradas, com cordas, em galhos de árvores a uma altura de cerca de 1,2 $\mathrm{m}$ do solo. Como iscas, foram utilizados $250 \mathrm{~g}$ de fígado bovino em decomposição, os quais eram colocados em um recipiente de plástico.

As armadilhas ficaram expostas a 50 metros do local de criação de suínos e do local de ordenha dos bovinos durante sete dias por mês, entre os dias 10 e 20 de cada mês. Retiraram-se os espécimes das armadilhas através de sacos plásticos de 60 litros, os quais eram posteriormente levados para o Laboratório de Biologia de Insetos para triagem, contagem e identificação. A identificação foi realizada com base na chave dicotômica das espécies (CARVALHO; RIBEIRO, 2000).

Os dados meteorológicos de temperatura média mensal e precipitação pluviométrica foram obtidos na Estação Agroclimatológica da Universidade Federal de Pelotas. Submeteram-se os resultados obtidos a análise de regressão polinomial, utilizando-se o programa estatístico SANEST (ZONTA; MACHADO, 1984).

\section{RESULTADOS E DISCUSSÃO}

Durante o período experimental, foram capturados 73 espécimens de $S$. chlorogaster, sendo que a distribuição da captura está demonstrada na Tabela 1. Na Figura 1, pode-se observar que a temperatura média mensal variou de $9,9{ }^{\circ} \mathrm{C}$ (julho) a $23,3{ }^{\circ} \mathrm{C}$ (março) e a precipitação pluviométrica acumulada mensal oscilou de 35,2 mm (abril) a 226,4 mm (junho). Na Figura 2, pode-se verificar que a velocidade média mensal do vento variou de $1,7 \mathrm{~m}^{-1} \mathrm{~s}^{-1}$ (abril) a 2,6 m.s ${ }^{-1}$ (outubro e janeiro). Levando-se em consideração a distribuição mensal dos 
califorídeos, constata-se que, nos 12 meses de coleta, houve uma variação mensal no número de espécies capturadas.

A presença desses califorídeos em criação de gado leiteiro pode estar associada à presença de matéria orgânica em decomposição, como cadáveres, resíduos de leite e de fezes, entre outros, visto que as larvas tem hábito necrótico e os adultos se adaptam bem ao ambiente modificado pelo homem (CARVALHO; MELLO-PATIU, 2008; LAOS et al., 2004; MALDONADO; CENTENO, 2003).

Tabela 1 - Ocorrência mensal de Sarconesia chlorogaster, capturadas por armadilha WOT, em criação de gado leiteiro, no período de março de 2007 a fevereiro de 2008, em Pelotas- RS.

Família/Espécies Meses (2007/2008)

$\frac{\varepsilon}{0}$

Mar Abr Mai Jun Jul Ago Set Out Nov Dez Jan Fev Total

Calliphoridae

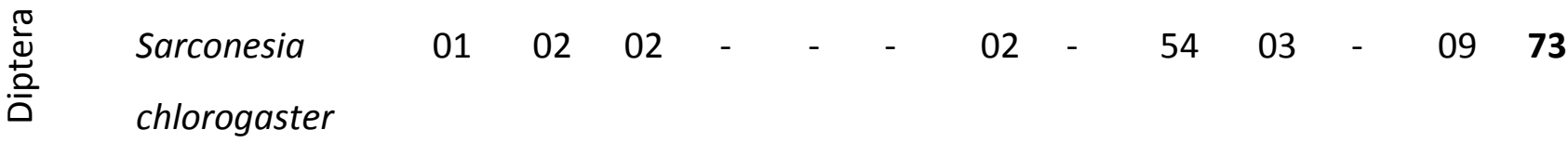

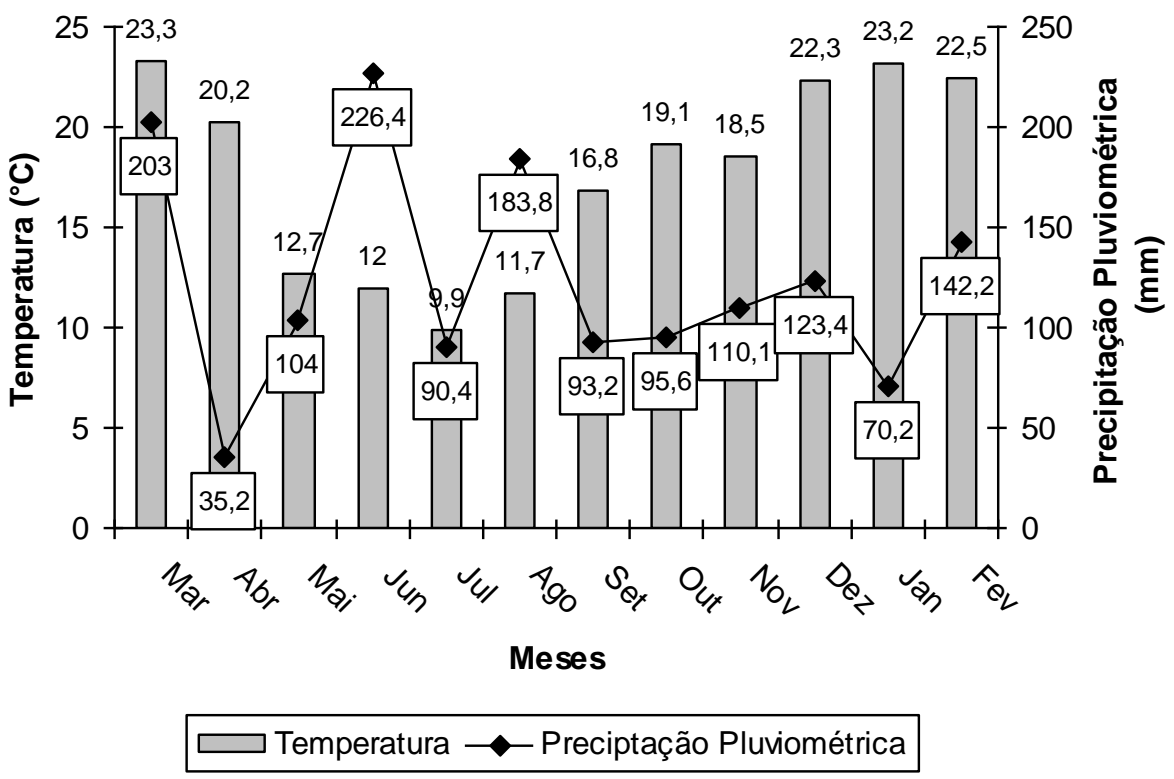

Figura 1 - Temperatura Média Mensal e Precipitação Pluviométrica, no período de março de 2007 a fevereiro de 2008, no sul do Rio Grande do Sul (Estação Agroclimatológica da Universidade Federal de Pelotas). 


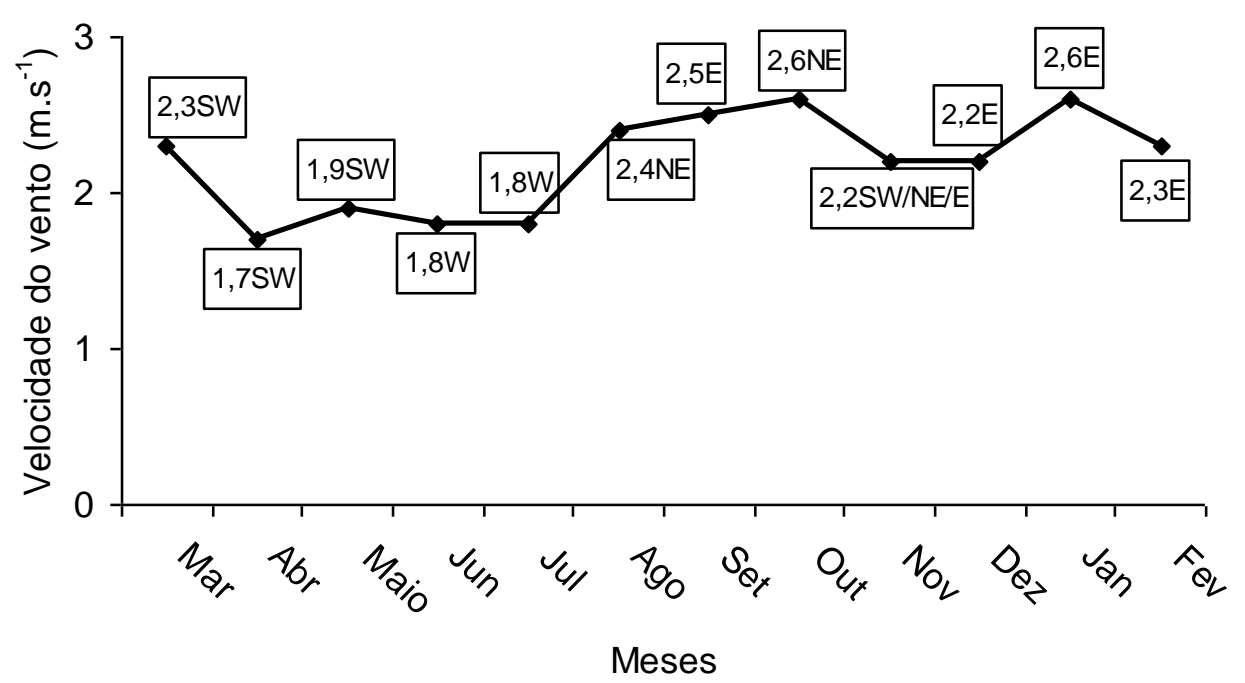

Figura 2 - Velocidade Média Mensal do vento, no período de março de 2007 a fevereiro de2008, no sul do Rio Grande do Sul (Estação Agroclimatológica da Universidade Federal de Pelotas).

S. chlorogaster apresentou pico populacional no mês de novembro (54), com temperatura média mensal de $18,5{ }^{\circ} \mathrm{C}$ e a menor ocorrência se deu no mês de março (1), em que a temperatura média mensal foi de $23,3{ }^{\circ} \mathrm{C}$. Nos meses de junho, julho, agosto, outubro e janeiro não houve captura dessa espécie (Tabela 1) (Figura 3).

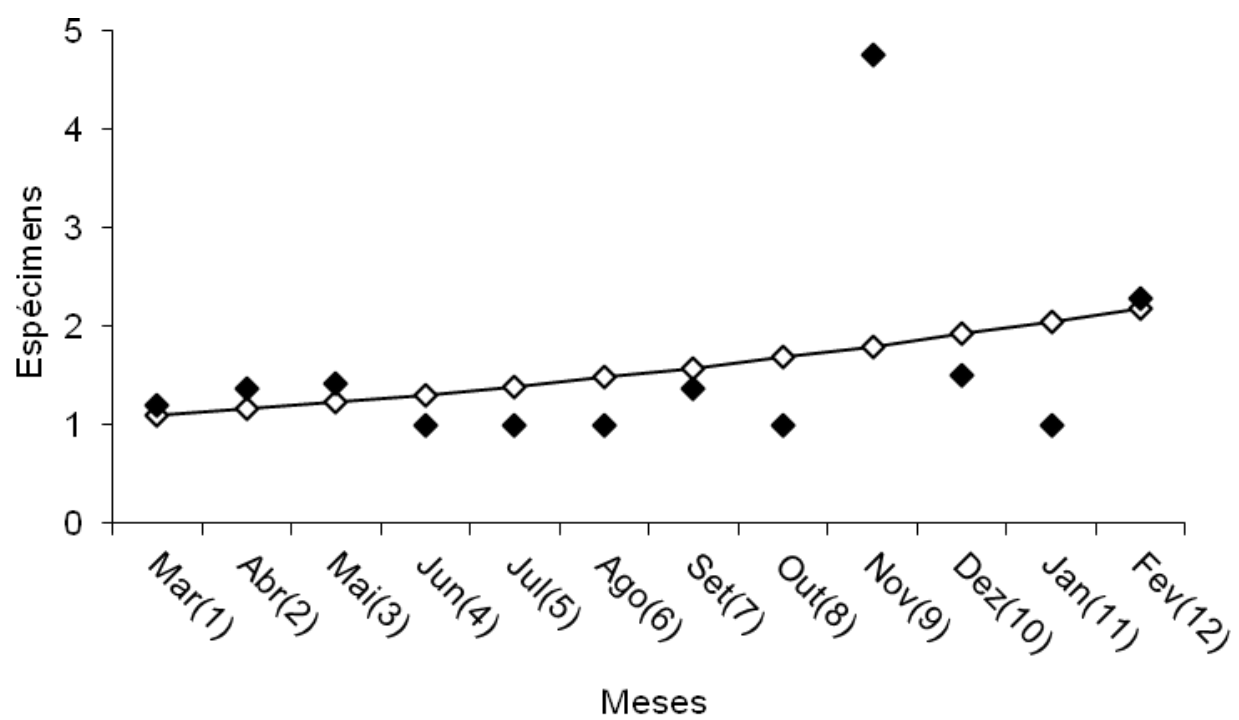

- Médias observadas $\prec$ Médias ajustadas

Figura 3 - Flutuação da população mensal de Sarconesia chlorogaster, capturadas em criação de gado leiteiro, no período de março de 2007 a fevereiro de 2008, em Pelotas- RS. 
Os resultados demonstram que houve uma variação mensal no número de espécimens do califorídeo capturado, sendo o mês de novembro o que apresentou o maior número de indivíduos capturados com uma combinação de boa temperatura média mensal $\left(18,5^{\circ} \mathrm{C}\right)$ e alta precipitação pluviométrica $(101,1 \mathrm{~mm})$. Temperaturas que propiciam menor intervalo entre gerações determinam altas densidades populacionais (GALLO et al., 2006).

Em trabalho realizado por Azevedo (2006), em Pelotas, RS, S. chlorogaster esteve presente de agosto a dezembro, com pico populacional nos meses de outubro e dezembro, diferentemente deste trabalho, cujo pico populacional ocorreu no mês de novembro.

Na cidade de Córdoba na Argentina, ao pesquisar a sazonalidade dos califorídeos, Horenstein et al. (2007), verificaram que S. chlorogaster esteve presente durante todo o experimento, mas foi particularmente abundante no inverno, o que difere do comportamento verificado neste estudo.

Entretanto, Krüger et al. (2010), avaliando a ocorrência de dípteros de importância forense, em carcaça de coelho doméstico, em Pelotas, RS, também verificaram a maior presença de S. chlorogaster no período do inverno.

Nos meses de junho, julho e agosto não houve captura, em virtude das baixas temperaturas, aliadas à alta precipitação pluviométrica. No mês de outubro, com o aumento das temperaturas médias mensais, tem início o restabelecimento da população, o que se reflete nos picos populacionais no verão. Já no mês de janeiro, a não captura de $S$. chlorogaster pode ter ocorrido em virtude da elevada temperatura, combinada com baixa precipitação pluviométrica, o que faz com que as fezes sequem mais rapidamente, inviabilizando ovos e larvas, conforme Tabela 1, Figuras 1 e 2. A não captura de indivíduos desta espécie nos meses de outubro e janeiro pode também estar relacionada com a maior velocidade média do vento nesses meses $\left(2,6 \mathrm{~m} . \mathrm{s}^{-1}\right)$, uma vez que o movimento exagerado da armadilha, com a força do vento, pode dificultar a localização do atrativo, diminuindo a entrada na armadilha, bem como dificultando a dispersão devido ao tamanho dos dípteros.

A análise de regressão polinomial indicou o modelo quadrático $(0,001 \%)$, como o que melhor se ajustou aos dados de flutuação populacional com $y_{i}=1,046451-0,0497408 x_{i}+$ $0,00377953 x_{i}^{2}$, onde $i=1,2, \ldots, 11,12$ (ordem dos meses do ano). Em biologia, esse modelo 
é bastante usado para explicar as relações entre variáveis que exprimem características de sistemas na natureza e que não são exatas, como neste experimento, em que as médias de captura oscilaram, durante o período experimental, principalmente em virtude da variação de temperatura.

Conforme Silveira-Neto (1976), os insetos mantém a temperatura corporal próxima à temperatura ambiente, a qual apresenta a capacidade de exercer influência direta (desenvolvimento e comportamento) e/ou indireta (alimentação) sobre os insetos.

\section{CONCLUSÃO}

De acordo com os resultados obtidos, nas condições deste trabalho, pode-se concluir que a ocorrência de S. chlorogaster em criação de bovinos leiteiros, na região sul do Rio Grande do Sul, é influenciada principalmente pela temperatura.

\section{OCCURRENCE OF Sarconesia chlorogaster (WIEDEMANN, 1830), IN DAIRY HERDS, IN THE SOUTH OF RIO GRANDE DO SUL, BRAZIL}

\section{ABSTRACT}

$\mathrm{T}$ he aim of the study was to provide information about the behavior of Sarconesia chlorogaster in a dairy cattle production, in the southern zone of the State of Rio Grande do Sul. This work was accomplished in the Centro Agropecuário da Palma, belonging to the Universidade Federal de Pelotas (UFPEL). For the capture of the calliphoridae wind oriented traps (W.O.T.) were used, $250 \mathrm{~g}$ of rotten liver beef was used as bait. The traps were set up 50 meters away from the milking site during seven days per month for 12 months; the captured specimens were removed from the traps between the $10^{\text {th }}$ and $20^{\text {th }}$ days and carried to the Insect Biology Laboratory for selection, count and identification. During the period from March 2007 to February 200873 S. chlorogaster specimens were captured. November had the most number of captures (53) and March was the month with less captures (01). An oscillation in the calliphoridae's population fluctuation was verified, mainly influenced by the variation of temperature. The presence of $S$. chlorogaster specimens in the southern zone of the State of Rio Grande do Sul is primarily influenced by the temperature.

Keywords: Traps. Behavior. Calliphoridae. 


\section{OCURRENCIA DE Sarconesia chlorogaster (WIEDEMANN 1830), EN GANADO DE LECHE EN EL SUR DE RIO GRANDE DO SUL, BRASIL}

\section{RESUMEN}

$\mathrm{E}$ ste estudio tuvo como objetivo proporcionar información sobre el comportamiento de Sarconesia chlorogaster en la producción de ganado lechero en la zona sur del estado de Rio Grande do Sul. El presente estudio se realizó en el Centro Agropecuario da Palma, el cual pertenece a la Universidade Federal de Pelotas (UFPEL). Para la captura de los califorideos, se usaron trampas de tipo W.O.T. (Wind Oriented Trap), cada una con $250 \mathrm{gr}$ de hígado de bovino como cebo en estado de descomposición. Las trampas fueron colocadas a 50 metros del local de ordeñe durante siete días por mes, a lo largo de 12 meses. Los ejemplares capturados, fueron retirados de las trampas entre el 10 y 200 día y llevados al Laboratorio de Biología de Insectos para su clasificación, conteo e identificación. Fueron capturados 73 ejemplares de S. chlorogaster durante el período de marzo de 2007 a febrero de 2008, donde en el mes de noviembre se obtuvo el mayor número de capturas (53) en contraste con el mes de marzo donde se obtuvo el menor número de capturas (01). De esta manera se observó una oscilación en la dinámica poblacional de este califorídeo, influenciado principalmente por la variación en la temperatura. La presencia de $S$. chlorogaster en el ganado lechero en la zona sur de estado de Rio Grande do Sul se ve influenciada principalmente por la temperatura.

Palabras clave: Trampa. Comportamiento. Calliphoridae.

\section{REFERÊNCIAS}

AZEVEDO, R. R. Calliphoridae (Insecta: Diptera) da região de Pelotas, Rio Grande do Sul, Brasil: Uma análise da dinâmica populacional. Pelotas: UFPEL, 2006. 47p. Monografia (Licenciatura e Bacharelado em Ciências Biológicas), Instituto de Biologia, Universidade Federal de Pelotas, 2006.

BONATTO, S. R. Ciclo de vida de Sarconesia chlorogaster (Wiedemann) (Diptera, Calliphoridae, Toxotarsinae), criada sob condições de laboratório em dieta artificial. Revista Brasileira de Zoologia, v. 13, p. 685-706, 1996.

BROCE, A. B.; GOODENOUGH, J. L.; COPPEDGE, J. R. A wind oriented trap for screw worm flies. Journal of Economic Entomology, v. 70, n. 4, p. 413-416, 1977.

CARVALHO, C. J. B.; RIBEIRO, P. B. Chave de identificação das espécies de Calliphoridae (Diptera) do sul do Brasil. Revista Brasileira de Parasitologia Veterinária, v. 9, p. 169-173, 2000. 
CARVALHO, C. J. B.; BORTOLANZA, M.; SILVA, M. C. C.; SOARES, E. D. G. Distributional patterns of the Neotropical Muscidae (Diptera). In: MORRONE, J. J.; LLORENTE, J. (Ed.) Una perspectiva latinoamericana de la Biogeografía. Mexico: Las Prensas de Ciencias, UNAM, 2003. p. 263-274.

CARVALHO, C. J. B; MELLO-PATIU, C. A. Key to the adults of the most common forensic species of diptera in South America. Revista Brasileira de Entomologia, v. 52, p. 390-406, 2008.

DEAR, J. P. A revision of the Toxotarsinae (Diptera, Calliphoridae). Papéis Avulsos de Zoologia, v. 32, n. 13, p. 145-182, 1979.

FURLANETTO, S. M. P.; CAMPOS, M. L. C.; HÁRSI, C. M. Microorganismos enteropatogênicos em moscas africanas pertencentes ao gênero Chrysomya (Diptera: Calliphoridae) no Brasil. Revista de Microbiologia, v. 15, n. 3, p. 170-174, 1984.

FURUSAWA, G. P.; CASSINO, P. C. R. Ocorrência e distribuição de Calliphotidae (Diptera, Oestroidea) em um fragmento de Mata Atlântica Secundária no Município de Engenheiro Paulo de Frontin, Médio Paraíba, RJ. Revista de Biologia e Ciências da Terra, v. 6, n. 1, p. 152-164, 2006.

GALLO, D.; NAKANO, O.; SILVEIRA-NETO, S.; CARVALHO, R. P. L.; BAPTISTA, G. C.; BERTI FILHO, E.; PARRA, J. R. P.; ZUCCHI, R. A.; ALVES, S. B.; VENDRAMIM, J. D.; MARCHINI, L. C.; LOPES, J. R. S.; OMOTO, C. Entomologia Agrícola. Piracicaba: FEALQ, 2006. 920p.

GREENBERG, B.; SZYSKA, M. L. Immature Stages and Biology of Fifteen Species of Peruvian Calliphoridae (Diptera). Annals of the Entomological Society of America, v. 77, p. 488-517. 1984.

HORENSTEIN, M. B.; LINHARES, A. X; ROSSO, B.; GARCÍA, M. B. Species composition and seasonal succession of Saprophagous calliphorids in a rural area of Córdoba: Argentina. Biological Research, v. 40, n. 2, p. 163-171, 2007.

JAMES, M. T. Family Calliphoridae. In: N. PAPAVERO, N. (Ed.). A catalogue of the Diptera of the Americas South of the United States. São Paulo, Museu de Zoologia da Universidade de São Paulo, 1970. Fascículo 102, p. 1-28.

KRÜGER, R. F.; KIRST, F. D.; DE SOUZA, A. S. B. Rate of development of forensically-important Diptera in southern Brazil. Revista Brasileira de Entomologia, v. 54, n. 4, p. 624-629, 2010.

LAOS, F.; SEMENAS, L.; LABUD, V. Factors related to the attraction of flies at a biosolids composting facility (Bariloche, Argentina). Science of the Total Environment, v. 328, p. 3340, 2004. 
MALDONADO, M. A.; CENTENO, N. Quantifying the Potential Pathogens Transmission of the Blowflies (Diptera: Calliphoridae). Memórias do Instituto Oswaldo Cruz, v. 98, p. 213-216, 2003.

MARILUIS, J. C.; LAGAR, M. C.; BELLEGARDE, E. J. Diseminación de enteroparasitos por Calliphoridae (Insecta: Oiptera). Memorias do Instituto Oswaldo Cruz, v. 84, p. 349-351, 1989.

MOURA, M. O.; BONATTO, S. R. Análise de sobrevivência e estimativa de entropia para Sarconesia chlorogaster (Wiedemann) (Diptera, Calliphoridae). Revista Brasileira de Zoologia, v. 16, p. 221-226, 1999.

OLIVEIRA, C. M. B. Biologia, flutuação populacional e patologia de Cochliomyia hominivorax (Coquerel, 1858). Rio de Janeiro: UFRRJ, 1980. 100p. Dissertação (Doutorado em Veterinária), Faculdade de Veterinária, Universidade Federal Rural do Rio de Janeiro, 1980.

QUEIROZ, S. M. P.; DE ALMEIDA, J. R.; DE CARVALHO, C. J. B.; DUDAS, L. Bionomia de Sarconesia chlorogaster (Wiedemann, 1830) (Diptera, Calliphoridae) em Curitiba, Paraná, Brasil. Anais da Sociedade Entomológica do Brasil, v. 14, p. 105-110, 1985.

SHERMAN, R. A.; HALL, M. J. R.; THOMAS, S. Medicinal maggots: an ancient remedy for some contemponary afflictions. Annual Review of Entomology, v. 45, p. 55-81, 2000.

SILVEIRA-NETO, S. Manual de Ecologia dos Insetos. São Paulo: Agronômica Ceres, 1976. 419p.

ZONTA, E. P.; MACHADO, A. A. Sanest-Sistema de Análise Estatística para Microcomputadores. Registrado na Secretaria Especial de Informática sob nํ․ 066060categoria A. Pelotas: Universidade Federal de Pelotas, 1984. 138p.

Autor para correspondência: Diego Moscarelli Pinto. Universidade Federal de Pelotas - Campus Universitário Capão do Leão. CEP 96010900 - caixa postal 354; Pelotas (RS)Brasil. dimoscarelli@yahool.com.br 